\title{
LA FIGURA DEL PROFESOR PIANISTA ACOMPAÑANTE EN LOS CONSERVATORIOS PROFESIONALES DE LA COMUNIDAD VALENCIANA
}

\author{
Elena Aguilar Gasulla
}

Conservatorio Superior de Música de Castellón

aguilargasulla@ hotmail.com

\begin{abstract}
Desempeñar la labor de profesor pianista acompañante en los conservatorios profesionales de música y danza de la Comunidad Valenciana suele convertirse en un verdadero desafio, ya que ni las leyes educativas han ofrecido posibilidad de formación específica al respecto, ni el trabajo con el profesor pianista acompañante se contempla como asignatura para los estudiantes de grado. Este estudio se centra en comprender cómo se desenvuelven estos profesionales, tanto desde el punto de vista personal como pedagógico, y qué repercusión tiene ese desafío en el desarrollo de sus identidades.

Assuming the role of piano accompanist teacher in professional music and dance conservatories of the Valencia Autonomous Community usually constitutes a real challenge, because educational laws have not offered any possibility of specific training, and it does not exist as a subject for piano majors. The purpose of this study is to understand how these professionals carry out their work, both from personal and pedagogical points of view, and what is the impact of this challenge in the development of their identities.
\end{abstract}

\section{INTRODUCCIÓN}

Este trabajo de investigación se centra en la figura del profesor pianista acompañante (PPA) y, concretamente, sobre el desafío que supone tener que desempeñar dicha función en el contexto de los conservatorios profesionales de música y danza de la Comunidad Valenciana. El objetivo del estudio es llegar a comprender la actividad y pensamiento profesional del PPA en unas determinadas circunstancias contextuales y temporales, tanto desde el punto de vista personal como pedagógico, y determinar los factores que pueden influir en "la manera" en que se asume esta función y aquellos que influyen en la "respuesta" que de ésta se deriva.

Siguiendo las reflexiones de Ocaña (2006) sobre el concepto de "desarrollo profesional", esta estrecha relación entre lo personal y lo profesional implica una evolución que se da de forma continuada en el tiempo y ayuda a interpretar los acontecimientos que se suceden a lo largo de toda la trayectoria laboral. Además, la comprensión de la actividad y conducta de estos profesionales requiere la comprensión de todos los contextos en los que interactúan y, a la vez, definen su labor.

Por una parte, trataré de especificar el contexto institucional o mesocontexto, descrito por Bresler (2004), trataré de especificar el contexto institucional o mesocontexto (estructura y fines del sistema escolar), su interacción con el microcontexto (intenciones y experiencia), y con el macrocontexto (valores de la cultura y sociedad). Por otra parte, y siguiendo a Eisner (1998), trataré de descubrir debajo de la conducta manifiesta el significado que los hechos tienen para quienes los experimentan en todos estos contextos e interacciones.

\section{JUSTIFICACIÓN Y CONTEXTO}

Al analizar las leyes y decretos que regulan (o deberían regular) las funciones del PPA en los conservatorios de música y danza en la Comunidad Valenciana, se observa que:

- Ni la Ley Orgánica de Educación 2/2006, de 3 de mayo (BOE nº 106, 4/5/2006), ni el Real Decreto 1577/2006, de 22 de diciembre, por el que se fijan los aspectos básicos del currículo de las enseñanzas profesionales de música hacen alguna referencia a la figura del PPA.

- El artículo 24 del Decreto 158/2007, de 21 de septiembre (DOCV n 5605, 25/9/2007), que regula el currículo de las Enseñanzas Profesionales de música en la Comunidad Valenciana, establece que: 
En los centros dependientes de la Conselleria competente en materia de educación, existirá la figura del Pianista Acompañante. Estos profesores se integrarán en el departamento o departamentos didácticos correspondientes a las especialidades en las que desempeñen su labor de apoyo.

- El artículo 25 del Decreto 156/2007, de 21 de septiembre (DOCV $n^{\circ}$ 5606, 25/09/07), que regula el currículo de las Enseñanzas Profesionales de danza de la Comunidad Valenciana establece que:

En los centros de enseñanza de danza, dependientes de la Conselleria competente en materia de educación, existirá la figura del Pianista Acompañante, Guitarrista Acompañante y Cantaor Acompañante. Estos profesores se integrarán en un departamento específico de contenido teórico musical, sin perjuicio de la obligación de asistir a los departamentos de las asignaturas en las que ejercen su actividad cuando sea solicitada su presencia.

- Ningún reglamento de organización y funcionamiento (ROF) de los conservatorios de música publicado hasta ahora hace referencia al desempeño de la labor de PPA, por lo que supondremos a priori que, a menos que algún conservatorio haya adoptado algún acuerdo interno al respecto, no queda delimitado el desempeño de sus funciones.

Si tenemos en cuenta las últimas convocatorias de oposiciones al cuerpo de Profesores de Música y Artes Escénicas publicadas en la Comunidad Valenciana a través de la Orden de 14 de abril de 2005 (DOCV no 4991, 22/04/05), Orden de 13 de marzo (DOCV n ${ }^{\circ}$ 5225, 23/03/06), Orden de 24 abril de 2007 (DOCV n ${ }^{\circ}$ 5501, 30/04/07), y Orden de 15 de abril de 2008 (DOCV no 5748, 23/04/08), en todas aparece la posibilidad de acceder a una plaza de piano, pero en ninguna de ellas hay opción de acceder a una plaza específica de PPA. Esto significa que, por una parte, en los conservatorios profesionales de música, cualquier persona que acceda a una plaza de piano puede desempeñar la función de PPA, ya que no existe una convocatoria específica de acceso y dicha figura debe "existir" en los conservatorios según los artículos arriba mencionados, y, por otra, que no obstante los alumnos de las diferentes especialidades tengan la posibilidad de trabajar con el PPA, dicha tarea o función no consta como asignatura en su plan de estudios.

Es importante tener en cuenta estos aspectos porque el hecho de que no esté regulada la función de PPA, y que ni siquiera conste como asignatura para los alumnos, contribuye a la marginación ${ }^{1}$ de este profesional, ya en segundo plano por la terminología que se le atribuye.

Este estudio se hace, por tanto, necesario para comprender mejor la función del PPA en los conservatorios de música, pero también en la sociedad, ya que mi experiencia pone en evidencia que se han generado una serie de prejuicios sobre esta figura, quizás creados y alimentados por un "imaginario colectivo" (Baeza, 2000; Borges-Rey, 2009; Cabrera, 2009), que influyen directamente sobre la manera de pensar y actuar de la persona que debe desempeñar esta labor.

\section{ESTADO DE LA CUESTIÓN}

Los estudios que se han llevado a cabo en este ámbito se han centrado en la figura del pianista acompañante (PA) ${ }^{2}$ en general, excepto el de Sanz (2003) que sitúa a este profesional en los conservatorios de música, tratándolo también desde la perspectiva de PPA. Algunos autores que tratan el aspecto de la terminología y los diferentes campos de acción del PA son: Abid-Mundim (2009),

\footnotetext{
${ }^{1}$ En la Enciclopedia Universal Ilustrada Europeo Americana (1980:320) se define el término "acompañante" como: "el que con la voz o con un instrumento acompaña a otro que toca otro instrumento musical o a uno o más cantantes". En el Diccionario Akal/Grove de la Música (2000:13) no aparece el término "acompañante", pero sí el de "acompañamiento" que se define como: "las partes subordinadas de una textura musical". Este mismo término es definido en el Gran Diccionario de la Lengua Española (2007:21) como: "sostén de la melodía de una voz o de un instrumento mediante la armonía que ejecuta en un instrumento secundario". Esta idea de parte subordinada o secundaria ha hecho que algunos estudiosos como Coelho (2003), Abid-Mundim (2009), Muniz (2009), Kacowicz (2011), hayan aportado sus alternativas a esta terminología, sugiriendo otros vocablos como "camerista", "co-repetidor" o "especialista".
}

${ }^{2}$ De ahora en adelante: PA. 
que también reflexiona sobre la formación empírica y académica, el trabajo de especialización con flauta travesera, y el disklavier como recurso tecnológico de apoyo a instrumentistas, y Muniz (2009), que individua siete diferentes funciones del pianista al que nunca se refiere como "acompañante".

Desde el punto de vista de la metodología cualitativa, cabe destacar los estudios de CoELHO (2003), que también reflexiona acerca de la terminología, la relación entre los conocimientos adquiridos durante el período de formación y las competencias específicas necesarias para desempeñar la función de PA, y Kokotsaki (2003, 2007), quien realiza un estudio acerca de las condiciones necesarias, desde la perspectiva del pianista, para conseguir una interpretación de alta calidad musical en un conjunto, centrándose en la búsqueda del equilibrio, estrategias de interacción (habilidades sociales, actitudes hacia el pianista), tiempo dedicado al estudio personal y a los ensayos de conjunto, desarrollo de habilidades específicas e integración con la audiencia a través del aspecto visual. Kubota (2009) estudia los cambios que se producen en los matices de "expresión" por parte del pianista durante una ejecución musical de conjunto, el proceso de interacción entre dos intérpretes, y analiza la diferencia del rol de co-ejecutante frente al de acompañante. Lehmann y Ericsson (1993) analizan los resultados de leer a primera vista entre pianistas expertos en acompañar y pianistas orientados a la ejecución de repertorio como solistas. Lehmann y Kopiez (2009) analizan la relación entre la percepción de la notación musical, lectura a vista y movimientos oculares. Siguiendo esta línea, Kacowicz (2011) dedica particular atención a la lectura primera vista, técnicas de transposición, memoria mnemónica y movimientos oculares en la lectura musical. Además, también reflexiona sobre la terminología utilizada en varios países. Lindo (1916) da indicaciones para desarrollar correctamente la habilidad de la lectura a vista y el transporte, analiza el papel marginal que sugiere la palabra "acompañante", los diferentes tipos de acompañamiento y situaciones, así como el temperamento necesario para ejercer de PA y adaptabilidad con diversos instrumentistas.

Volviendo al estudio de Sanz (2003), su investigación se lleva a cabo en el Conservatorio Profesional de Música Arturo Soria de Madrid, del que estudia la normativa a la que está vinculado, la organización de las asignaturas, así como la situación de los alumnos y profesores. Además, propone una serie de objetivos que deberían añadirse a la asignatura de instrumento y una metodología para la impartición de los contenidos y una correcta temporización; reflexiona sobre los espacios adecuados para el correcto desarrollo de las clases (pianos de buena calidad y afinados), y sobre cómo debería llevarse a cabo la evaluación. Sólo este trabajo trata la figura del PPA, es decir, la perspectiva de profesor. Todos los demás estudios abordan esta figura desde la perspectiva del PA y sus variadas terminologías, pero, de entre todas las que se mencionan: co-repetidor, colaborador, acompañador, acompañante, especialista, coach y camerista, ninguna más hace referencia a ésta como docente.

Esto es relevante para este estudio en cuanto que significa que los contextos en los que se han llevado a cabo las anteriores investigaciones difieren considerablemente de éste por no considerar todas las variables y posibilidades desde el punto de vista de la profesión, y su interacción en la multiplicidad de contextos a los que está ligado.

Por otra parte, este estudio ha tomado como guía principal los trabajos de Bolívar (2002 y 2006), Csikszentmihaly (2008), Hargreaves (1996), Marchesi (2007), Napione-Bergé (2008), Vaello (2009), para comprender e interpretar algunas categorías de datos, relacionadas con las identidades emergentes a partir de la información analizada. En particular, el trabajo de Bolívar (2006) ha resultado fundamental para entender cuáles son los factores principales que influyen en la formación de la identidad personal y profesional de los docentes. En su trabajo apunta que la identidad es un elemento crucial en los modos cómo los profesores construyen cotidianamente la naturaleza de su trabajo (motivaciones, satisfacción y competencias) y señala que la identidad docente se construye y reconstruye mediante las interacciones sociales que los profesores tienen en los contextos particulares en que actúan. Los trabajos de Csikszentmihaly (2008) han resultado particularmente interesantes porque relaciona conceptos como habilidades y desafíos personales, acción y percepción, etc., y en ellos emergen elementos recurrentes en los otros estudios, pertinentes con el concepto y desarrollo de 
identidad profesional. El trabajo de Hargreaves (1996) se centra en la postmodernidad como condición social que abarca determinadas pautas de relaciones sociales, económicas, políticas y culturales. Entre ellas, confiere determinada importancia a los conflictos que pueden plantearse entre el control burocrático y la potenciación profesional de los profesores como factores influyentes en la realización de sus identidades individuales.

Marchesi (2007), también se refiere a la identidad profesional en general, como algo que no se adquiere al incorporarse a una determinada actividad laboral, sino como un largo proceso de experiencias vividas, de encuentros con los otros y de reflexión sobre la propia práctica. Además, también reflexiona sobre el reconocimiento social, las fases y ciclos del desarrollo profesional, sobre el bienestar y malestar docente, las emociones y relaciones del profesorado. Napione-Bergé (2008) realiza un análisis y estudio sobre la salud del docente y los factores implicados en su buen mantenimiento, especialmente los psicosociales. Algunas cuestiones que desarrolla son: el estrés laboral, el síndrome de quemarse por el trabajo en el profesorado, el desempeño de roles, relaciones interpersonales, etc. En su estudio, Vaello (2009), propone romper el desequilibrio que ha existido secularmente en la educación a favor de los aspectos cognitivos respecto de los socioemocionales a los que atribuye la máxima importancia. Según el autor, es la madurez socioemocional la que nos permite superar situaciones complicadas y conseguir logros de largo alcance, para lo cual considera necesario desarrollar toda una serie de competencias sociales y emocionales. Algunos de los argumentos principales en los que se centra son: las actitudes y, en particular, la actitud resiliente, los conflictos interpersonales, la ansiedad, la gestión del tiempo, la autoestima, las habilidades del docente, los diferentes roles, las conductas que se derivan, etc.

Teniendo en cuenta todos estos aspectos, la finalidad de este estudio ha sido descubrir cómo se integran todas las facetas que constituyen la identidad de estos profesionales, y de qué manera es cuestionada y modificada en un contexto concreto, pero también "como proceso de socialización, en espacios sociales de interacción, mediante identificaciones y atribuciones, donde la imagen de sí mismo se configura bajo el reconocimiento de otros" (Bolívar, Fernández, \& Molina, 2005:3).

\section{METODOLOGÍA Y DISEÑO}

Las preguntas principales de este estudio son:

1. ¿Qué desafío representa asumir la función de PPA, tanto desde el punto de vista personal como pedagógico, y cómo influye la formación recibida en las necesidades profesionales?

2. ¿Qué tipo de relación/interrelación existe con los profesores tutores y alumnos a los que acompaña, tanto a nivel personal como pedagógico, de qué manera la perciben y cómo afecta a su actividad?

3. ¿Cómo influye el deber asumir tal desafío, la formación recibida, las relaciones personales y profesionales sobre su identidad como pianistas, y en qué medida incurren en un conflicto de roles?

La investigación se aborda, en conjunto, a través de la metodología cualitativa. Según Strauss y Corbin (1998), por "investigación cualitativa" se entiende cualquier tipo de investigación que lleva a descubrimientos no basados en procedimientos estadísticos o de otro tipo de cuantificación. La principal técnica utilizada son las entrevistas biográficas, a partir de las cuales se obtienen unos relatos que "re-construyo" de forma narrativa en las historias de vida. Durante el proceso de preparación y desarrollo de las entrevistas se han tenido en cuenta las indicaciones y sugerencias aportadas por Stake (1998), Kvale (2011), Bresler (2004), y Bresler y Stake (1992). He reconstruido los relatos de los sujetos desde este enfoque biográfico-narrativo, utilizando la herramienta "historias de vida" ya que, según la definición de Giménez-Béliveau y Mallimaci (2011:1):

...la historia de vida investiga sobre un individuo determinado donde se incluye su propio relato y es complementado por el investigador con otros documentos...

Por este motivo, esta herramienta me ha parecido la más adecuada para relacionar los valores personales y culturales con el desarrollo de la profesión, y me ha permitido desarrollar un informe 
final comparativo donde emergen las identidades de cada uno de los informantes, a partir del cual he podido llegar a unas conclusiones.

Otras técnicas de recogida de datos que se han utilizado han sido las observaciones no participantes, siguiendo a Eisner (1998) y Stake (1998), notas de campo, dibujos, y análisis de documentos como el currículum académico, currículum profesional, programas de audiciones y acuerdos internos de regulación de funciones.

El criterio principal para la elección definitiva de los tres casos que conforman este estudio piloto ha sido la búsqueda de tres perfiles diferentes contrastantes. Para ello, empecé por un muestreo intencionado o teórico a través de voluntarios, tal como sugieren Martín-Crespo y Salamanca (2007:2), y avancé hacia un muestreo deliberado basándome en las necesidades de información para esta investigación. La elección final es la siguiente:

\begin{tabular}{|c|c|c|}
\hline & Pasado & Presente (2011-12) \\
\hline $\begin{array}{l}\text { Informante } 1^{3} \\
\text { (funcionario) }\end{array}$ & $\begin{array}{l}\text { - PPA en Conservatorio música: canto. } \\
\text { Asignación forzosa - interino } \\
\text { - PPA en Conservatorio danza } \\
\text { Asignación forzosa - interino } \\
\text { - PPA en Conservatorio música: violín, viola, } \\
\text { violonchelo, contrabajo, flautas, clarinetes, } \\
\text { oboes, fagots. } \\
\text { Asignación forzosa - interino }\end{array}$ & $\begin{array}{l}\text { - PPA en Conservatorio música: } \\
\text { contrabajo y viola } \\
\text { Elección voluntaria - (funcionario) + profesor } \\
\text { piano + profesor de música de cámara }\end{array}$ \\
\hline $\begin{array}{l}\text { Informante } 2 \\
\text { (funcionario) }\end{array}$ & $\begin{array}{l}\text { Ninguna experiencia como PPA en } \\
\text { conservatorios }\end{array}$ & $\begin{array}{l}\text { - PPA Conservatorio música: Canto } \\
\text { Elección voluntaria. } \\
\text { Profesor de piano + profesor "acompañamiento" }\end{array}$ \\
\hline $\begin{array}{l}\text { Informante } 3 \\
\text { (interino) }\end{array}$ & $\begin{array}{l}\text { - PPA en Conservatorio música: violines, } \\
\text { violas, trompas, tubas, bombardinos, } \\
\text { trompetas, oboes, fagots, flautas, clarinetes. } \\
\text { Asignación forzosa } \\
\text { - PPA en Conservatorio de música: viento- } \\
\text { metal } \\
\text { Asignación forzosa } \\
\text { - PPA en Conservatorio de música: Canto, } \\
\text { Asignación forzosa cuerda. }\end{array}$ & En bolsa de trabajo \\
\hline
\end{tabular}

Tabla 1: Perfiles y experiencias de los informantes definitivos

Por lo que respecta al análisis de datos, he utilizado, por una parte, algunos argumentos y conceptos predeterminados directamente relacionados con las preguntas de la investigación, pero también han surgido otros nuevos a raíz de la categorización de los datos, por lo que se ha trabajado tanto de manera deductiva como inductiva. Para esta etapa de análisis y trascripción de los datos he utilizado el software de análisis de datos cualitativo Atlas.ti, de esta manera, el trabajo de clasificación y agrupación de conceptos por categorías ha resultado bastante ordenado, en cuanto el programa permite, al mismo tiempo, la jerarquización de todos los datos.

Siguiendo a Bolívar, Fernández y Molina (2005), he analizado cada entrevista desde dos puntos de vista diferentes: por una parte, como descripción de cada caso con entidad propia (análisis vertical o diacrónico), y, por otra, desde una perspectiva transversal (horizontal o sincrónica) con las ocho categorías que había determinado, componentes de la identidad. El primer análisis ha permitido la "re-construcción" de las historias de vida de los sujetos desde la narración autobiográfica y, el

\footnotetext{
${ }^{3}$ De ahora en adelante: I1, I2, I3.
} 
segundo, la elaboración de un informe final conjunto, que concluye con los resultados de las identidades emergentes.

Por lo que respecta a los criterios de calidad, y siguiendo a Stake (1998), se han utilizado los siguientes:

- Triangulación de las fuentes de datos: he tratado de observar si el sujeto sigue siendo el mismo en otros momentos o espacios, en la interactuación con otras personas. Para ello, la información recogida durante las entrevistas se ha intentado cuestionar a través de observaciones no participantes durante las clases y audiciones, análisis de los materiales (como acuerdos internos de regulación de las funciones del PPA), y legislación.

- Triangulación de los informantes: ésta ha resultado de especial importancia por ayudar a contrastar datos emergentes de las interrelaciones. En particular, he realizado algunas entrevistas a alumnos, a profesores tutores de las especialidades con las que trabaja el PPA, y otros profesores-compañeros del centro. También he tenido en cuenta los datos recogidos en algunas entrevistas de los informantes finalmente descartados, por la cercanía profesional y vinculación al mismo contexto.

- Triangulación metodológica: los enfoques múltiples dentro de un único estudio como la observación no participante, las entrevistas biográficas, su contraste con sucesivas preguntas y otras sesiones de entrevista, la revisión de la documentación, el diario de campo, y la comparación de los datos de los tres sujetos en el informe final, me han permitido afianzar la confianza en mi interpretación.

- Triangulación del observador (con otros investigadores externos). En relación a este último punto, y en medida de lo posible, se ha seguido la recomendación sugerida por Aróstegui y Rusinek (2010) de generar o inserirse en alguna red de "amigos críticos", como evaluadores de la calidad y apoyo para la mejora de nuestros trabajos. Por otra parte, Stake (1998) también considera una buena estrategia presentar las observaciones (con nuestra interpretación o sin ella) a otros investigadores o expertos para que aporten interpretaciones alternativas.

\section{ANÁLISIS DE DATOS}

A partir del análisis de datos han emergido los siguientes elementos o "categorías" comunes a todos los informantes:

- Formación, desarrollo de habilidades.

- Experiencias (Retos-desafíos, motivación, expectativas futuro).

- Salud y estrés laboral.

- Interrelaciones (Actitudes)

- Percepción del rol

- Reconocimiento social

- Marginación

- Regulación

En cuanto a la formación, Bolívar (2006) habla de la importancia de la trayectoria biográfica individual que determina la construcción de un saber profesional propio, fruto de una trayectoria particular. Siguiendo al autor, realizo una comparación de los diferentes itinerarios formativos: 


\begin{tabular}{|l|l|l|l|}
\cline { 2 - 4 } \multicolumn{1}{c|}{} & \multicolumn{1}{|c|}{ I1 } & \multicolumn{1}{c|}{ I2 } & \multicolumn{1}{c|}{ I3 } \\
\hline \multirow{5}{*}{$\begin{array}{l}\text { Formación } \\
\text { oficial }\end{array}$} & $\begin{array}{l}\text { Título Profesor Piano } \\
\text { (Decreto 2618/1966) }\end{array}$ & $\begin{array}{l}\text { Grado Medio } \\
\text { Título Profesor Piano } \\
\text { (Decreto 2618/1966) }\end{array}$ & $\begin{array}{l}\text { Grado Medio } \\
\text { Título Profesor Piano } \\
\text { (Decreto 2618/1966) }\end{array}$ \\
\cline { 2 - 5 } & $\begin{array}{l}\text { Estudios superiores } \\
\text { Decreto 2618/1966) }\end{array}$ & $\begin{array}{l}\text { Título Superior Piano ABRSM de } \\
\text { Londres } \\
\text { (Taylor, 1982) }\end{array}$ & $\begin{array}{l}\text { Estudios superiores } \\
\text { Grado Superior LOGSE, } \\
\text { Esp. Pedagogía del Piano } \\
\text { (Ley 1/1990) }\end{array}$ \\
\cline { 2 - 5 } & $\begin{array}{l}\text { Otras titulaciones } \\
\text { Título Profesor teoría-solfeo, } \\
\text { repentización y acompañamiento } \\
\text { (Decreto 2618/1966) }\end{array}$ & $\begin{array}{l}\text { Otras titulaciones } \\
\text { Título de Maestro en Educación } \\
\text { Musical } \\
\text { (Decreto 1440/1991) }\end{array}$ & Otras titulaciones \\
\hline
\end{tabular}

Tabla 2: Itinerario formativo de cada informante.

Bolívar (2006) afirma que dentro de la identidad personal, ocupa un lugar relevante el trabajo o profesión, de ahí la relevancia de la identidad profesional. En este estudio, la primera experiencia en un conservatorio se revela particularmente importante, ya que es el primer afrontamiento de la realidad profesional, superados los estudios que conducen a la obtención del Título Superior.

En la siguiente tabla damos muestra de cuál fue la experiencia que supuso el mayor desafío, o situación de mayor dificultad, para cada uno de los informantes:

\begin{tabular}{|c|c|c|c|}
\cline { 2 - 4 } \multicolumn{1}{c|}{} & I1 & I2 & I3 \\
\hline & 2005-06: & PPA: & Cuerda \\
& Danza clásica. & Reto personal & (excepto contrabajo); \\
Experiencias: & Jornada completa. & & viento-madera \\
El desafío & Curso completo. & & (excepto saxos); \\
& 2006-07: & & viento-metal. \\
& Cuerda, viento - madera & & Jornada completa. \\
& (excepto saxos). & & 2 semanas \\
& Jornada completa. & & (final curso). \\
\hline
\end{tabular}

Tabla 3: Situación de mayor dificultad-estrés en sus años de experiencia

Al analizar las experiencias como PA fuera de los conservatorios de música, se observa que, el hecho de ser situaciones en las que pueden elegir asumir dicha función o no, hace que su motivación sea diferente. Esto se debe a que, por una parte, ninguna administración se lo impone y no están sometidos a ningún tipo de presión que condicione su situación laboral, y, por otra parte, en todos los casos, representa una ganancia económica adicional:

\begin{tabular}{|c|c|c|c|c|}
\hline & & I1 & $\mathrm{I} 2$ & I3 \\
\hline \multirow{4}{*}{$\begin{array}{l}\text { Experiencias } \\
\text { fuera de los } \\
\text { conservatorios }\end{array}$} & $\begin{array}{c}\text { Durante } \\
\text { formación }\end{array}$ & Cantantes & $\begin{array}{l}\text { Canto } \\
\text { Chelo }\end{array}$ & $\begin{array}{c}\text { Coros } \\
\text { Viento-metal }\end{array}$ \\
\hline & Motivación & Amistad & Hedonismo & Económica \\
\hline & $\begin{array}{c}\text { Períodos de } \\
\text { trabajo en } \\
\text { conservatorios }\end{array}$ & $\begin{array}{c}\text { Canto } \\
\text { Cuerda } \\
\text { Guitarra }\end{array}$ & $\begin{array}{l}\text { Saxofón } \\
\text { Trompeta }\end{array}$ & $\begin{array}{c}\text { Coros } \\
\text { Viento-metal }\end{array}$ \\
\hline & Motivación & Económica Amistad & $\begin{array}{c}\text { Económica } \\
\text { Hedonismo } \\
\text { Prestigio }\end{array}$ & Económica \\
\hline
\end{tabular}


Tabla 4: Experiencias individuales como PA fuera de los conservatorios

Estas experiencias también influyen decisivamente en el grado de motivación para querer desempeñar o no el rol de PPA como profesión, lo cual tiene que ver, tal como afirma Bolívar (2006), con las expectativas futuras del individuo que representan los futuros estados o situaciones deseadas:

\begin{tabular}{|c|c|c|c|}
\cline { 2 - 4 } \multicolumn{1}{c|}{} & I1 & I2 & I3 \\
\hline Expectativas & Intención de continuidad & Intención de continuidad & Sin posibilidad de elección \\
\hline Condicionantes & $\begin{array}{c}\text { Mismas o similares años } \\
\text { anteriores }\end{array}$ & Solo misma especialidad & Interinidad \\
\hline Motivación & Cursos bajos & $\begin{array}{c}\text { Hedonismo } \\
\text { Inversión personal }\end{array}$ & $\begin{array}{c}\text { Ampliar repertorio: cursos } \\
\text { altos }\end{array}$ \\
\hline
\end{tabular}

Tabla 5: Expectativas, condicionantes y motivación para seguir desempeñando la función de PPA en el futuro

A modo de resumen, se puede establecer una "etiqueta" o título final que refleja lo que ha supuesto el desarrollo personal y profesional en cada trayectoria individual y evolución de las experiencias vividas (hasta ahora), en su rol como PPA:

\begin{tabular}{|c|c|c|c|}
\hline & I1 & $\mathrm{I} 2$ & I3 \\
\hline Evolución & $\begin{array}{c}\text { Del exceso... } \\
\text { al aburrimiento }\end{array}$ & Inversión personal & $\begin{array}{l}\text { El reto: cantidad repertorio } \\
\text { nuevo - límite tiempo }\end{array}$ \\
\hline
\end{tabular}

Tabla 6: Evolución de la trayectoria individual de cada informante

Todos los informantes han manifestado sufrir algún problema de salud durante su experiencia como PPA, tanto desde el punto de vista físico como psicológico:

\begin{tabular}{|c|c|c|c|}
\cline { 2 - 4 } \multicolumn{1}{c|}{} & I1 & I2 & I3 \\
\hline Salud y estrés laboral & Estrés & $\begin{array}{c}\text { Estrés - ansiedad } \\
\text { Cabeza saturada } \\
\text { Desgaste físico } \\
\text { Problemas espalda }\end{array}$ & $\begin{array}{c}\text { Problemas espalda } \\
\text { Prarar }\end{array}$ \\
\hline
\end{tabular}

Tabla 7: Problemas de salud durante las experiencias como PPA

Por otra parte, Vaello-Orts (2009:19), describe las competencias socioemocionales como:

...el conjunto de habilidades que permiten interactuar con los demás o con uno mismo de forma satisfactoria, además de contribuir a la satisfacción interna, a la consecución de éxitos personales y profesionales, y a una adecuada adaptación al contexto.

Es precisamente este tipo de interrelaciones, personales y profesionales, que constituyen el análisis de este apartado:

\begin{tabular}{|c|c|c|c|c|}
\hline & & I1 & I2 & I3 \\
\hline \multirow[t]{2}{*}{ INTERRELACIONES } & Presente & Apoyo moral & \multirow[b]{2}{*}{ La comunicación } & Subordinación \\
\hline & Pasado & Subordinación & & $\begin{array}{c}\text { vs } \\
\text { respeto }\end{array}$ \\
\hline
\end{tabular}

Tabla 8: Interrelaciones personales y profesionales en los centros de trabajo

Tal como afirma Marchesi (2007), se puede observar que las emociones provocan tendencias de acción: todas las actitudes derivadas de las experiencias de los tres informantes, así como el grado de 
implicación en el rol de PPA, están directamente relacionadas con las interrelaciones en el contexto de trabajo (compañeros, alumnos, directivos). Sigue una tabla comparativa:

\begin{tabular}{|c|c|c|c|c|}
\hline & & I1 & $\mathrm{I} 2$ & I3 \\
\hline \multirow[b]{2}{*}{ Actitudes } & Presente & Colaborativa & \multirow{2}{*}{$\begin{array}{c}\text { Colaborativa } \\
\uparrow \\
\text { Flexibilidad }\end{array}$} & \multirow{2}{*}{$\begin{array}{c}\text { Adaptativa } \\
\uparrow \\
\text { Superación }\end{array}$} \\
\hline & Pasado & Neutra & & \\
\hline
\end{tabular}

Tabla 9: Actitudes de colaboración derivadas de las interrelaciones.

Napione-Vergé (2008:31) define la ambigüedad de rol como "el grado de incertidumbre que el sujeto que desempeña un rol tiene respecto al mismo". Dentro de la asunción de este rol como PPA, se producen algunas diferencias en cuanto al grado de percepción que cada informante tiene del desempeño de su labor:

\begin{tabular}{|c|c|c|c|}
\hline & I1 & $\mathrm{I} 2$ & I3 \\
\hline ¿PA o PPA? & PPA (Cursos bajos) & $\begin{array}{c}\text { PA y PPA (Según el nivel } \\
\text { del alumno) }\end{array}$ & PA y PPA \\
\hline $\begin{array}{l}\text { Identificación al interno del } \\
\text { departamento de piano }\end{array}$ & $\begin{array}{l}\text { Música de cámara } \\
\text { (no único rol) }\end{array}$ & $\begin{array}{c}\text { Combinación de } \\
\text { diversos roles: } \\
\text { piano, acompañamiento, } \\
\text { PPA, evolución de la } \\
\text { interpretación }\end{array}$ & $\begin{array}{l}\text { Indiferente } \\
\text { (no único rol) }\end{array}$ \\
\hline
\end{tabular}

Tabla 10: Ambigüedad de rol

Bolívar (2006) afirma que un elemento crucial en la configuración de la identidad profesional, es el reconocimiento social que tiene, ya que se construye con las identidades que los otros reconocen. En las siguientes tablas comparativas se analizan la percepción del reconocimiento y marginación del rol por cada informante:

\begin{tabular}{|c|c|c|c|}
\hline & I1 & $\mathrm{I} 2$ & I3 \\
\hline Reconocimiento social & $\begin{array}{c}\text { En función de sus } \\
\text { habilidades } \\
(\text { danza }) \\
+ \\
\text { aplauso audiciones, nombre } \\
\text { en programa }\end{array}$ & $\begin{array}{c}\text { En función de sus } \\
\text { habilidades } \\
\text { (improvisación) } \\
+ \\
\text { solicitud opinión } \\
+ \\
\text { elogios públicos } \\
+ \\
\text { aplauso audiciones, nombre } \\
\text { en programa }\end{array}$ & $\begin{array}{c}\text { En función de sus } \\
\text { habilidades } \\
\text { (rapidez) } \\
+ \\
\text { Abrir tapa piano }\end{array}$ \\
\hline
\end{tabular}

Tabla 11: Percepción del reconocimiento social como PPA

El escenario de una audición es el lugar de exposición a la sociedad, experiencia que contribuye a la construcción de la identidad profesional del PPA, ya que, tal como afirman Bolívar, Fernández y Molina (2005:3):

La identidad para sí, como proceso biográfico, reclama complementarse, como proceso social y relacional, con la confirmación por los otros de la significación que el actor otorga a su identidad. La identidad personal se configura, como una transacción recíproca (objetiva y subjetiva), entre la identidad atribuida por otros y la identidad asumida. 
En este sentido, el hecho de abrir la tapa del piano, contribuye a no sentirse en segundo plano, a proyectar una imagen de protagonismo sobre el escenario, de la que esperan recibir un cierto grado de valoración social, lo cual contribuirá directamente a fortalecer su identidad profesional.

\begin{tabular}{|c|c|c|c|}
\hline & I1 & I2 & I3 \\
\hline Marginación & $\begin{array}{c}\text { Relación profesores tutores } \\
\text { No evaluable } \\
\text { Falsos mitos }\end{array}$ & $\begin{array}{c}\text { Divismo (profesores y } \\
\text { alumnos) } \\
\text { Impuntualidad } \\
\text { No evaluable } \\
\text { Instalaciones y mantenimiento } \\
\text { Administración }\end{array}$ & $\begin{array}{c}\text { Relación profesores y alumnos } \\
\text { Desorganización centro } \\
\text { Recursos }\end{array}$ \\
\hline
\end{tabular}

Tabla 12: Percepción de la marginación social como PPA

Finalmente, todos aportan sus propuestas de regulación:

\begin{tabular}{|c|c|c|c|}
\hline & I1 & I2 & I3 \\
\hline $\begin{array}{l}\text { Sugerencias para la } \\
\text { regulación del rol de PPA }\end{array}$ & $\begin{array}{c}\text { Asignatura evaluable } \\
+ \\
\text { Repartir la tarea entre varias } \\
\text { personas del departamento }\end{array}$ & $\begin{array}{c}\text { Sustitución o ampliación al } \\
\text { "aprendizaje colaborativo" } \\
\text { entre alumnos }\end{array}$ & $\begin{array}{c}\text { Bolsa específica de PPA } \\
+ \\
\text { Repartir la tarea }\end{array}$ \\
\hline
\end{tabular}

Tabla 13: Propuestas para la regulación de la figura de PPA

Estas categorías principales me han servido para hacer un estudio comparativo de los elementos más importantes que han conducido al desarrollo personal y profesional los tres sujetos, y establecer los aspectos emergentes de las diferentes identidades. La evolución de estas identidades a través de las diferentes experiencias muestra cómo en un mismo individuo pueden darse características opuestas (pesimismo-optimismo, inseguridad-seguridad) según la fase de su desarrollo personal y profesional que atraviesa.

\begin{tabular}{|c|c|c|c|}
\hline & $\mathrm{I} 1$ & $\mathrm{I} 2$ & I3 \\
\hline Identidades & $\begin{array}{c}\text { culpabilidad, vergüenza, } \\
\text { inseguridad, } \\
\text { sufrimiento, } \\
\text { frustración, } \\
\downarrow \\
\text { pesimismo } \\
\downarrow \\
\text { voluntad superación } \\
\downarrow \\
\text { responsabilidad } \\
\downarrow \\
\text { autoafirmación, atribución } \\
\text { importancia rol PPA } \\
\downarrow \\
\text { optimismo } \\
\downarrow \\
\text { seguridad } \\
\downarrow \\
\text { elección voluntaria } \\
\downarrow\end{array}$ & $\begin{array}{c}\text { seguridad } \\
\downarrow \\
\text { elección voluntaria } \\
\downarrow \\
\text { inversión personal } \\
\downarrow \\
\text { orgullo, } \\
\text { honor } \\
\downarrow \\
\text { prestigio } \\
\downarrow \\
\text { reconocimiento social } \\
\downarrow \\
\text { disponibilidad de trabajo } \\
\text { extra }\end{array}$ & $\begin{array}{c}\text { ganas de llorar, } \\
\text { inseguridad, } \\
\text { pesimismo } \\
\downarrow \\
\text { aceptación sobrecarga } \\
\text { trabajo } \\
\downarrow \\
\text { a la defensiva } \\
\downarrow \\
\text { responsabilidad } \\
\downarrow \\
\text { respeto } \\
\downarrow \\
\text { reconocimiento } \\
\downarrow \\
\text { autoafirmación } \\
\downarrow \\
\text { optimismo } \\
\downarrow \\
\text { voluntad superación } \\
\downarrow\end{array}$ \\
\hline
\end{tabular}




\begin{tabular}{|c|c|c|c|}
\hline & $\begin{array}{c}\text { no disponibilidad trabajo } \\
\text { extra } \\
\downarrow \\
\text { aburrimiento }\end{array}$ & & seguridad \\
\hline
\end{tabular}

Tabla 14: Características y evolución de las identidades

Estos resultados me han llevado a relacionar la fase de desarrollo profesional que atraviesa cada informante con las identidades emergentes, según las etapas apuntadas por Marchesi (2007), identidades que vienen condicionadas por una multiplicidad de factores en una intersección, por una parte, del itinerario y contexto formativo y profesional; y, por otra, a través de la evolución y afrontamiento de diversas situaciones a nivel personal y pedagógico, la reflexión y autoafirmación, donde la personalidad individual de cada uno se revela fundamental a la hora de afrontar y percibir, pero también, otorgar un valor o determinar una actitud.

\section{DISCUSIÓN Y CONCLUSIONES}

Sin ninguna voluntad de establecer generalizaciones, expongo el resultado de un análisis comparativo a partir de la información obtenida. Para ello, he intentado dar respuesta a las preguntas de partida de esta investigación.

1. ¿Qué desafío representa asumir el rol de PPA, tanto desde el punto de vista personal como pedagógico, y cómo influye la formación recibida en las necesidades profesionales?

Concluyo que desempeñar el rol de PPA en la red de conservatorios de la Comunidad Valenciana ha supuesto (y sigue suponiendo) un verdadero desafío para los informantes que han participado en este estudio.

Para dos de ellos representó la primera experiencia con la realidad en el mundo profesional tras haberse presentado a un concurso-oposición en la especialidad de piano. El hecho de acceder a una vacante/sustitución en régimen de interinidad, significaba que no tenían posibilidad de elección respecto a la elección de esta función. Así pues, esta asignación conllevó un cierto impacto emocional: por una parte, no se veían cumplidas las expectativas de lo que esperaban del mundo profesional, ya que ellos se habían formado en la especialidad de "piano", habían opositado para ser profesores de "piano", y habían sido contactados para cubrir una plaza de "piano", y, por otra parte, la formación recibida a través de sus itinerarios formativos no les había dado la posibilidad de desarrollar las habilidades necesarias para desempeñar esta función exitosamente, al no existir ninguna asignatura ni especialidad directamente relacionada con la figura del PPA, como profesión.

Además, estos elementos personales se entrecruzaban con el factor profesional, en cuanto que tenían que satisfacer unas demandas para un centro y unos alumnos, que se concretaban en el estudio de una gran cantidad de repertorio para diversos instrumentos y piano, que ninguno de ellos conocía o había estudiado previamente, por ser, precisamente, su primera experiencia.

El desconocimiento de esta función, el sentimiento de una carencia de habilidades específicas y la presión asociada al factor tiempo en vista a las audiciones, hizo que esta primera experiencia representara la de mayor estrés y presión de toda su carrera (hasta el momento de las entrevistas). Si, además, a todos estos factores les añadimos el hecho de que se dan en un contexto legislativo donde el desempeño de esta función no está regulado, se hacen evidentes grandes problemas de desorganización, aumenta la sensación de estrés, y aparece el sentimiento de estar "al servicio de" compañeros, alumnos y directivos. Sentimiento que puede asociarse al de "subordinación" que, superado el primer impacto o shock inicial y tomada conciencia del estado real de su situación, les llevó a un cambio de actitud, a una voluntad de superación: afrontarlo como un desafío personal. En lugar de renunciar a la plaza, negociar la cantidad de repertorio o quejarse por la sobrecarga de trabajo, prefirieron adoptar una actitud resiliente, es decir, resistir de manera activa y creadora (Vaello-Orts, 
2007), ya que el miedo a perder el trabajo o a que les echaran de la bolsa de profesores interinos, hizo que estuvieran dispuestos a soportar determinadas condiciones que incluso llegaron a ocasionarles serios problemas de salud física y mental.

Esta manera de afrontar la situación, esta actitud de voluntad por salir "airoso" de la misma, les ha permitido, no solo haber desarrollado habilidades específicas necesarias para ejercer como PPA, sino, a la vez, salir fortalecidos emocionalmente de las mismas, y mejor preparados para afrontar una nueva situación, un nuevo reto. Nuevos desafíos que aún pueden llegar, como en el caso de uno de los informantes al que llamaron para una vacante en un conservatorio de danza, y que pueden representar experiencias aún más estresantes y angustiantes, si no se conoce la jerga específica y si no se ha recibido formación que desarrolle las habilidades de improvisación, principalmente requeridas para ejercer de PA en los conservatorios de danza.

En este estudio también hemos visto el caso contrario de otro informante que, siendo ya funcionario de carrera, tardó años en poder elegir el rol de PPA; lo asumió también como reto personal, de forma voluntaria y consciente, apenas se le presentó la ocasión, pero en el sentido de inversión personal, y no como reacción a una imposición.

2. ¿Qué tipo de relación/interrelación existe con los profesores tutores y alumnos a los que acompaña, tanto a nivel personal como pedagógico, de qué manera la perciben y cómo afecta a su actividad?

La calidad de las interrelaciones genera unas actitudes en respuesta que terminan siendo diplomáticas y colaborativas, ya que son principalmente los "juicios" de sus compañeros de trabajo los que más influyen en su percepción de reconocimiento del rol.

Su preocupación sobre "cómo son vistos" en el centro puede deberse a la inseguridad de ejercer una función que nunca antes han ejercido como profesión, por lo que el reconocimiento según la evolución del desarrollo de sus habilidades es crucial para su afirmación e identidad como pianistas, vistos los factores que pueden contribuir a cuestionarla en el mismo contexto. En este sentido, el desafío personal cobra doble sentido, ya que la satisfacción generada a través de su superación contribuye a la propia afirmación de identidad personal y profesional.

3. ¿Cómo influye el deber asumir tal desafío, la formación recibida, las relaciones personales y profesionales sobre su identidad como pianistas, y en qué medida incurren en un conflicto de roles?

Ninguno de los tres informantes había recibido formación oficial específica para poder ejercer la función de PPA; han sido las experiencias personales previas a la inserción en el mundo laboral las que les han hecho "tomar conciencia" de la existencia de dicha figura, tanto en el contexto laboral como fuera de él. La falta de formación específica y de regulación se revelan, pues, como las fuentes más importantes de estrés y marginación del rol, que, además contribuyen a poner en entredicho su identidad como pianistas.

El grado de percepción de su función varía según el nivel de los alumnos: durante los primeros cursos, sienten que su función es más pedagógica, es decir, se identifican más como PPA, en cambio, a medida que los alumnos aumentan de nivel, se sienten más implicados en el "hecho musical", como PA.

Por lo que respecta al rol o función que pueden desempeñar al interno del departamento de piano, los tres coinciden en la voluntad de variabilidad de funciones. En este sentido, aunque el hecho de asumir el rol de PPA como primera experiencia en un centro pueda poner en entredicho su identidad por no cumplir con las expectativas deseadas, después de haber superado dicha experiencia, y a medida que se van integrando en el contexto profesional, todos los informantes demuestran una inclinación por la variedad de roles, lo cual representa un progreso en su desarrollo personal y profesional que, tal como afirma Ocaña (2006), es un constructo en continua evolución. 


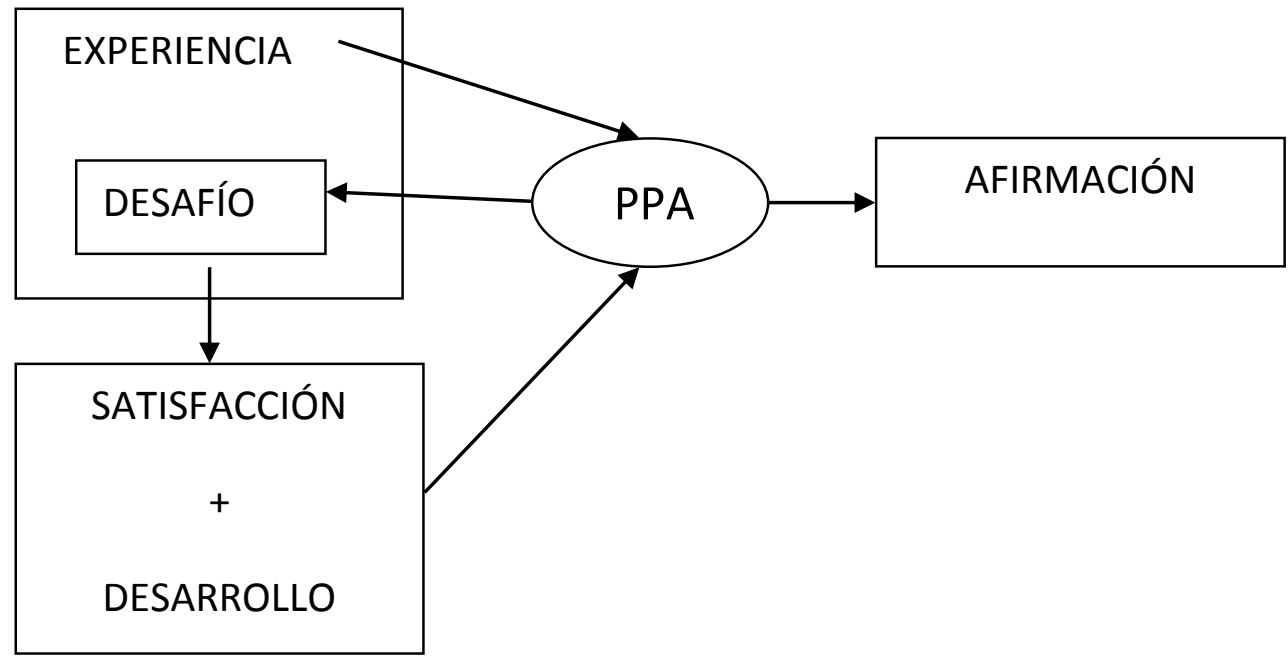

Figura 1: Proceso de afirmación de la identidad como PPA

Algunos de los elementos emergentes comunes a los tres informantes son:

- Carencia de formación específica para desempeñar el rol de PPA

- Importancia de la influencia que ejercen las experiencias vividas previamente a la entrada en el contexto laboral que, de alguna manera, determinan una cierta preferencia hacia determinados instrumentos.

- Voluntad de continuidad bajo un mínimo de condiciones de regulación para evitar la sobrecarga de trabajo y estrés.

- Trascendencia de las relaciones con los profesores tutores de las especialidades que acompañan, tanto desde el punto de vista pedagógico de colaboración y mejora de la calidad de la clase para el alumno, como desde el punto de vista personal, que también influye en su motivación de continuidad.

- Interferencia de lo público con lo privado a través de la manifestación de problemas de salud y estrés laboral derivados de la actividad profesional.

- La generación de ciertas actitudes en respuesta a las emociones generadas a través de las interrelaciones (alumnos, profesores, directivos).

- Tendencia a evitar conflictos en las interrelaciones.

- Propensión a desarrollar una actitud colaborativa y de flexibilidad, en condiciones de respeto y reconocimiento hacia la labor del PPA.

- Percepción de un desdoblamiento de rol: en la faceta de pianista y en la de profesor, porcentaje que varía según el nivel de los alumnos.

- Percepción de reconocimiento en función de sus habilidades y desenvoltura en el contexto profesional.

- Preferencia de combinación de diversos roles en la distribución de jornada completa, lo cual es sinónimo de dinamismo y versatilidad.

- Poca importancia atribuida a la terminología como causa de su reconocimiento/marginación.

- Falta de regulación y no participación en la evaluación como causas de marginación del rol.

- La contribución de la función de PPA a su desarrollo personal y profesional, y consolidación de su identidad.

Otros elementos emergentes son:

- Dificultad para relacionarse con los cantantes por su divismo.

- Dificultad para relacionarse e integrarse en los conservatorios de danza.

- Diferencia de motivaciones para aceptar experiencias fuera del contexto laboral: por amistad, 
hedonismo, prestigio y, en último lugar, por necesidad económica.

- Inclusión del nombre del PPA en los programas de las audiciones y solicitud de aplauso específico para el PPA al finalizar las mismas como muestra de reconocimiento hacia su labor.

- Simbolismo de la apertura de la tapa del piano como señal de importancia y autoreconocimiento.

- Falsos mitos relativos a la figura del PPA que contribuyen, por una parte, a una determinada "visión" de este colectivo entre músicos, estudiantes y otros profesores, y, por otra, a su marginación.

- Elementos físicos del lugar del trabajo (ruidos, vibraciones, limpieza instalaciones, insonorización adecuada de las aulas...) calidad y afinación de los instrumentos como elementos de marginación.

Solo queda por confiar en la temprana regulación de este rol, el de PPA como profesión, para que se disipen todos los prejuicios y falsos mitos entorno a esta figura y sea integrada y reconocida plenamente por sus habilidades y competencias. A este respecto se da la paradoja de que es casualmente en el Decreto 158/2007, citado anteriormente, donde se hace referencia al pianista como PA, no como PPA, cuando, por otra parte, es la misma administración la que convoca un concursooposición para acceder a la docencia en la especialidad de piano, a cuyo departamento pertenece la figura que ellos mismos no consideran como "docente" en la legislación.

Un primer paso se ha dado a través de la publicación de la Orden 24/2011, de 2 de noviembre, de la Conselleria de Educación, Formación y Empleo, por la que se establecen y autorizan los planes de estudio de los centros de enseñanzas artísticas superiores de música dependientes del ISEACV, conducentes a la obtención del título de Graduado o Graduada en Música que, finalmente, establece y regula como asignatura la materia de "Repertorio de Lied/Acompañamiento instrumental".

El hecho de que se reconozca como asignatura dentro de un plan de estudios, después de tantos años, contribuirá a colmar parte de ese "vacío" legal existente que debería culminar con el reconocimiento de la labor de PPA como especialidad y, finalmente, como profesión.

\section{Referencias}

Abid-Mundim, A. (2009). Pianista colaborador: A formação e atuação performática voltada para o acompanhamento de flauta transversal. Tesis de máster. Universidade Federal de Minas Gerais. Consultado el 11/2/12 en http://www.bibliotecadigital.ufmg.br/dspace/bitstream /1843/AAGS7XMLVZ/1/o_pianista_colaborador.pdf

Aróstegui, J.L., \& Rusinek, G. (2010). Haciendo amigos (y enemigos): Qué es y qué no es investigación en educación musical. En G. Rusinek, M. E. Riaño, \& N. Oriol (Eds.), Actas del Seminario Internacional en Educación Musical 2010 (pp.16-19). Madrid: Sociedad para la Educación Musical del Estado Español - Universidad Complutense de Madrid.

Baeza, M. A. (2000). Los caminos invisibles de la realidad social: Ensayo de sociología profunda sobre los imaginarios sociales. Santiago: Sociedad Hoy - RIL.

Bolívar, A. (2002). “¿De nobis ipsis silemus?”: Epistemología de la investigación biográficonarrativa en educación. Revista Electrónica de Investigación Educativa, 4(1). Consultado el 10/3/2012 en http://redie.uabc.uabc.mx/vol4no1/contenido-bolivar.html

Bolívar, A. (2006). La identidad profesional del profesorado de secundaria: Crisis y reconstrucción. Málaga: Aljibe.

Bolívar, A., Fernández, M. \& Molina, E. (2005). Investigar la identidad profesional del profesorado: Una triangulación secuencial. Forum: Qualitative Social Research Sozialforschung, 6(1), Art. 12. Consultado el 10/3/2012 en http://www.qualitative-research.net/index.php/fqs/article/view /516/1117 
Borges-Rey, E. L. (2009). Imaginario colectivo musical: Convenciones en el proceso de interpretación del sentido de la música. Icono, 14, 210-231. Consultado el 11/2/12 en http://www.icono14.net/revista/num14/14_icono14_eddyborges.pdf

Bresler, L. (2004). Metodología de investigación cualitativa: Prestando atención a la música escolar como género en sus micro y macro contextos. Revista Electrónica Complutense de Investigación en Educación Musical, 1(1), 1-18. Consultado el 25/2/12 en http://revistas.ucm.es/index.php/ RECI/article/view/RECI0404110001B

Bresler, L., \& Stake, R. (1992). Qualitative research methodology in music education. En R. Colwell (Ed), Handbook of research on music teaching and learning (pp.75-90). Nueva York: Schirmer.

Cabrera, D. (2009). Imaginario social, comunicación e identidad colectiva. Institut de la Comunicació -Universidad Autónoma de Barcelona. Consultado el 15/2/12 en http://www.portalcomunicacion.com/dialeg/paper/pdf/143_cabrera.pdf

Coelho, M. A. C. (2003). Pianista acompanhador: Um estudo analítico de suas competências e ações enquanto produtor musical. Anais do XIV Congresso da ANPPOM. Porto Alegre, 2003. Consultado el 15/2/12 en http://www.anppom.com.br

Conselleria de Educación y Cultura (2005). Orden de 14 de abril de 2005, de la Conselleria de Cultura, Educación y Deporte, por la que se convocan procedimientos selectivos de ingreso, accesos y adquisición de nuevas especialidades a los cuerpos docentes de profesores de Enseñanza Secundaria, profesores de Escuelas Oficiales de Idiomas, profesores de Música y Artes Escénicas, profesores de Artes Plásticas y Diseño y profesores técnicos de Formación Profesional. Valencia: Diario Oficial de la Comunidad Valenciana, 4991, 22/4/05.

Conselleria de Educación y Cultura (2006). Orden de 13 de marzo de 2006, de la Conselleria de Cultura, Educación y Deporte, por la que se convocan procedimientos selectivos de ingreso, accesos y adquisición de nuevas especialidades a los cuerpos docentes de profesores de Enseñanza Secundaria, profesores de Escuelas Oficiales de Idiomas, profesores de Música y Artes Escénicas, profesores de Artes Plásticas y Diseño y profesores técnicos de Formación Profesional. Valencia: Diario Oficial de la Comunidad Valenciana, 5225, 23/03/06.

Conselleria de Educación y Cultura (2007). Orden de 24 de abril de 2007, de la Conselleria de Cultura, Educación y Deporte, por la que se convocan procedimientos selectivos de ingreso, accesos y adquisición de nuevas especialidades en los cuerpos docentes de profesores de Enseñanza Secundaria, profesores de Escuelas Oficiales de Idiomas, profesores de Música y Artes Escénicas y profesores técnicos de Formación Profesional. Valencia: Diario Oficial de la Comunidad Valenciana, 5501, 30/04/07.

Conselleria de Educación y Cultura (2008). Orden de 15 de abril de 2008, de la Conselleria de Educación, por la que se convocan procedimientos selectivos de ingreso, accesos y adquisición de nuevas especialidades en los cuerpos docentes de profesores de Enseñanza Secundaria, profesores de Escuelas Oficiales de Idiomas, profesores de Música y Artes Escénicas y profesores técnicos de Formación Profesional. Valencia: Diario Oficial de la Comunidad Valenciana, 5748, 23/04/08.

Conselleria de Educación y Cultura (2007). Decreto 156/2007, de 21 de septiembre por el que se establece el currículo de las enseñanzas profesionales de danza y se regula el acceso a estas enseñanzas. Valencia: Diario Oficial de la Comunidad Valenciana, 5606, 25/09/07.

Conselleria de Educación y Cultura (2007). Decreto 158/2007, de 21 de septiembre, por el que se establece el currículo de las enseñanzas profesionales de música y se regula el acceso a estas enseñanzas. Valencia: Diario Oficial de la Comunidad Valenciana, 5606, 25/9/2007.

Conselleria de Educación, Formación y Empleo. (2011). Orden 24/2011, de 2 de noviembre, de la Conselleria de Educación, Formación y Empleo, por la que se establecen y autorizan los planes de estudio de los centros de enseñanzas artísticas superiores de música dependientes del ISEACV, conducentes a la obtención del título de Graduado o Graduada en Música. Valencia: Diario Oficial de la Comunidad Valenciana, 6648, 10/11/2011. 
Csikszentmihalyi, M. (2008). Flow, the psychology of optimal experience. Nueva York: Harper.

Csikszentmihaly, M. (2008). El yo evolutivo: Una psicología para un mundo globalizado. Barcelona: Kairós.

Eisner, E. W. (1998). El ojo ilustrado: Indagación cualitativa y mejora de la práctica educativa. Barcelona: Paidós.

Enciclopedia Universal Ilustrada Europeo Americana (Vol. II). (1980). Madrid: Espasa-Calpe.

Giménez-Béliveau, V. \& Mallimaci, F. (2006). Historias de vida y método biográfico. En Estrategias de Investigación cualitativa (pp. 175-212). Barcelona: Gedisa.

Gran Diccionario de la Lengua Española (3ª ed). (2007). Barcelona: Larousse.

Hargreaves, A. (1996). Profesorado, cultura y postmodernidad. Madrid: Morata.

Kacowicz, A. T. (2011). O pianista co-repetidor e a arte do acompanhemento: Seu significado e importância no cenário musical atual. Tesis de máster. Universidade Federal do Rio Janeiro. Consultado el 16/2/12 en http://www.docpro.com.br/escolademusica/bibliotecadigital.html

Kokotsaki, D. (2003). A grounded theory approach to understanding the pianist's perspective on the attainment of high quality in musical ensemble performance. Tesis doctoral inédita. University of Sheffield.

Kokotsaki, D. (2007). Understanding the ensemble pianist: A theoretical framework. Psychology of Music, 35(4), 641-668. doi: 10.1177/0305735607077835

Kubota, Y. (2009). The nature of professional accompanists and their roles: Performing with musical excellence and enjoying communicative interaction. En A. Williamon, S. Pretty, \& R. Buck (Eds.), International Symposium on Performance Science 2009. Londres: AEC-Centre for Performance Science-Royal College of Music. Consultado el 18/2/12 en http://www.legacyweb.rcm.ac.uk/cache/f10020010.pdf

Kvale, S. (2011). Las entrevistas en investigación cualitativa. Madrid: Morata.

Lehmann, A. C., \& Ericsson, K. A. (1993). Sight-reading ability of expert pianists in the context of piano accompanying. Psychomusicology, 12, 182-195. Consultado el 6/2/12 en http://blade2.vre.upei.ca/ojs/index.php/psychomusicology/article/viewFile/699/806\#page=88

Lehmann, A. C., \& Kopiez R. (2009). Sight-reading. En S. Hallam, I. Cross, \& M. Thaut (Eds.), The Oxford handbook of music psychology (pp. 344-351). Oxford: Oxford University Press.

Lindo, A. (1916). The art of accompanying ( $3^{\mathrm{a}}$ ed.). Nueva York: Schirmer.

Marchesi, A. (2007). Sobre el bienestar de los docentes: Competencias, emociones y valores. Madrid: Alianza.

Martín-Crespo, C. \& Salamanca, A. (2007). El muestreo en la investigación cualitativa. Departamento de Investigación de FUDEN. Nure Investigación. Consultado el 21/2/12 en http://bibliocomunidad.com/web/libros/FMetodologica_27.pdf

Ministerio de Educación y Ciencia (1990). Ley Orgánica 1/1990, de 3 de octubre, de Ordenación General del Sistema Educativo. Madrid: Boletín Oficial del Estado. Núm. 238, 4/10/90. Consultado el 22/06/12 en http://www.boe.es/boe/dias/1990/10/04/pdfs/A28927-28942.pdf

Ministerio de Educación y Ciencia (1991). Real Decreto 1440/1991, de 30 de agosto, por el que se establece el título universitario oficial de Maestro, en sus diversas especialidades y las directrices generales propias de los planes de estudios conducentes a su obtención. Madrid: Boletín Oficial del Estado, 244, 11/10/1991.

Ministerio de Educación y Ciencia (1966). Decreto 2618/1966 de 10 de septiembre, sobre Reglamentación general de los Conservatorios de Música. Madrid: Boletín Oficial del Estado, 254, 24/10/66.

Ministerio de Educación y Ciencia (2006). Ley Orgánica de Educación 2/2006, de 3 de mayo, de Educación. Madrid: Boletín Oficial del Estado, 106, 04/05/2006. 
Ministerio de Educación y Ciencia (2006). Real Decreto 1577/2006, de 22 de diciembre, por el que se fijan los aspectos básicos del currículo de las enseñanzas profesionales de música reguladas por la Ley Orgánica 2/2006, de 3 de mayo, de Educación. Madrid: Boletín Oficial del Estado, $18,20 / 1 / 2006$.

Muniz, F. R. S. (2010). O pianista camerista, correpetidor e colaborador: As habilidades nos diversos campos de atuação. Universidade Federal de Goiás. Consultado el 11/2/12 en http://portais.ufg.br/uploads/270/original_FranklinMuniz.pdf

Napione-Bergé, M. E. (2008). ¿Cuándo se “quema” el profesorado de secundaria? Madrid: Díaz de Santos.

Ocaña, A. (2006). Desarrollo profesional de las maestras de educación musical desde una perspectiva biográfico-narrativa. Revista Electrónica Complutense de Investigación en Educación Musical, 3 (3), (pp. 1-14). Consultado el 25/2/12 en http://revistas.ucm.es/index.php/RECI/article /view/RECI0606110003A

Ocaña, A. (2006). Identidad y ciclos de desarrollo profesional de los maestros y maestras de educación musical. Tesis doctoral. Universidad de Granada. Consultado el 07/06/12 en http://hdl.handle.net/10481/1357

Sadie, S. (2000). Diccionario Akal/Grove de la música. Madrid: Akal.

Sanz, R. (2001). El profesor de instrumento y el profesor pianista acompañante. Música y Educación 14(4), 45-62.

Stake, R. (1998). Investigación con estudio de casos. Madrid: Morata.

Strauss, A.; Corbin, J. (1998). Basics of qualitative research: Techniques and procedures for developing grounded theory ( $2^{\mathrm{a}}$ ed.). Thousand Oaks (California): Sage.

Taylor, C. (1982). These music exams. A guide to ABRSM exams for candidates, teachers and parents. Londres: The Associated Board of the Royal Schools of Music. Consultado el 23/06/10 en http://www.abrsm.org/resources/theseMusicExams0607.pdf

Vaello-Orts, J. (2009). El profesor emocionalmente competente. Barcelona: Graó.

Manuscrito recibido el 20/11/2012 y evaluado anónimamente. Aceptado para su publicación el 13/11/2013. 